\title{
Estrategia de enseñanza a través de diferentes campos de conocimiento en primer curso del grado en arquitectura técnica
}

\author{
Q. Anguloa, M. Pons ${ }^{b}$, A. García ${ }^{c}$ y M.E. Torner ${ }^{d}$ \\ a-b-c-d Universidad Politécnica de Valencia (e-mail: quianib@csa.upv.es)
}

\begin{abstract}
The higher education area needs new methodological implementations that facilitate active learning.

Therefore, this text aims to demonstrate the experience developed in the project of Educational Innovation and Improvement in the subjects of construction and economy teached on first semester of the first year of the Degree in Technical Architecture (UPV), for the development of a Business Plan in the Construction sector.

The methodology used is based on activities for projects, so that the student uses the acquired knowledge actively to the development of a project interconnecting the competency of the different subjects.

The results show that this methodology has allowed the interdisciplinary knowledge of both subjects. This allows watertight knowledge a priori of the two subjects, can be understood and work together, assimilating and applying the same concepts. Thanks to that, the student performance has been much positive due to prior knowledge on the subject to be treated and its direct application.
\end{abstract}

Keywords: Competences, transversal, evaluation, interdisciplinary

\footnotetext{
Resumen

El área de la educación superior necesita nuevas implementaciones metodológicas que facilitan el aprendizaje activo.

El presente documento pretende demostrar la experiencia desarrollada en el proyecto de innovación educativa y mejora, en las materias de construcción I $y$ economía en el primer semestre del primer curso del Grado en Arquitectura Técnica (UPV), para el desarrollo de un Plan de Negocios en el sector de la construcción.

La metodología utilizada se basa en las actividades de los proyectos, de manera que el alumno utiliza los conocimientos adquiridos de forma activa al desarrollo de un proyecto de interconexión de la competencia de los diferentes temas.

Los resultados muestran que esta metodología ha permitido el conocimiento interdisciplinario de ambas materias. Esto permite que el conocimiento hermético "a priori" de las dos asignaturas, se puede entender y trabajar de
} 
Estrategia de enseñanza a través de diferentes campos de conocimiento en primer curso del grado en arquitectura técnica

manera conjunta, asimilando y aplicando los mismos conceptos. Gracias a ello, el rendimiento de los estudiantes ha sido más positivo debido a los conocimientos previos en la materia a tratar, y de su aplicación directa.

Palabras clave: Competencias, transversalidad, evaluación, interdisciplinariedad.

\section{Introducción}

El adaptar la metodología de educación universitaria al proyecto de Espacio Europeo de Educación Superior, requiere de proyectos de innovación y mejora docentes. El trabajo interdisciplinar entre distintas áreas de conocimiento y profesorado con unos objetivos comunes, permite experimentar nuevos modelos de formación para la docencia universitaria (UPV, EICE 2015).

En este sentido, las metodologías basadas en problemas de aprendizaje tienen una larga trayectoria. El aprendizaje basado en problemas (ABP) sugiere un método de enseñanza en el que los estudiantes aprenden a través de la resolución de un problema dado, en el que puede tener más de una solución correcta. Mediante un trabajo en grupo, los estudiantes colaboran para identificar lo que necesitan para solucionar el problema (Hmelo-Silver, 2004). Esto no es una tarea sencilla pues puedes encontrarte con diferentes dificultades. Algunos autores trabajan sobre el problema y la autoeficacia (Dunlap, 2005). Aunque la adquisición de conocimiento y habilidades hace posible que se conozca el rendimiento, sin la autoeficacia de la actuación no puede ni siquiera intentarse (entendemos eficacia como capacidad de lograr el efecto que se desea o se espera). Elementos como la colaboración, la reflexión y el planteamiento de los problemas auténticos de la vida real, hacen mejorar la autoeficacia de los alumnos. De este modo puede ir aumentando progresivamente los niveles de autoeficacia de los alumnos. Otras líneas de experimentación están basadas en las relaciones entre las características de problemas, conductas relacionadas con el rendimiento y el rendimiento académico en el aprendizaje (Sockalingam et al, 2011). Uno de los problemas planteados en la actualidad es el grado de conocimiento del problema. Autores como Sockalingam (Sockalingam and Schmidt, 2013) muestran en su investigación la relación entre la familiaridad de problemas y el aprendizaje de los estudiantes en cursos basados en PBL. La familiaridad entendida como problema, se refiere a la medida en que un problema encaja con los conocimientos y experiencias previas de los estudiantes. En este sentido la familiaridad, en el sentido positivo, ayuda al aprendizaje de los alumnos en la materia enfrentada al problema, pero la falta de familiaridad puede dar lugar a más preguntas, el pensamiento y el razonamiento, cosa que tampoco ha de valorarse como negativa.

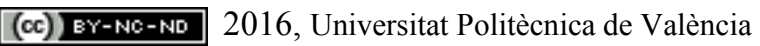




\section{Objetivos}

Siguiendo con los dos planteamientos destacados anteriormente, cabria extrapolar el aprendizaje con sistemas PBL en el primer curso de Grado en Arquitectura Técnica. Por un lado se pone en práctica el sentido del conocimiento del problema (familiaridad) y por otro lado el rendimiento académico vinculado a la autoeficacia del alumno. De este modo, el equipo de innovación y calidad educativa, denominado "Actividades Transversales en primer curso de Ingeniería de la Edificación" considera que el desarrollo de competencias transversales proporciona al alumno una formación integral. En el curso 2015/2016, se plantea un Proyecto de Innovación y Mejora Educativa entre las asignaturas de Construcción I y Economía del primer curso de Grado en Arquitectura Técnica. Siendo asignaturas de departamentos distintos y contenidos estancos, no existe una interrelación de conocimientos a la hora de abordar las prácticas de construcción, con contenidos de economía y viceversa, dificultando el aprendizaje y la adquisición de conocimientos.

En este documento se presentan los resultados obtenidos de esta experiencia entre las dos asignaturas, favoreciendo la adquisición y desarrollo de las competencias transversales. Así mismo se constata que la interdisciplinariedad ayuda a la metodología PBL favoreciendo la familiaridad del problema, la autoeficacia del alumno y, por lo tanto, desembocando en un mejor rendimiento. La percepción de sus resultados académicos de los estudiantes, muestran un claro éxito frente a los alumnos que no han formado parte de esta investigación. Por otra parte el profesorado participante de la experiencia de Aprendizaje Basado en Problemas, confirma la relación entre las contribuciones que los estudiantes hacen al proceso de grupo tutorial basado en problemas, como es observado de manera positiva por sus compañeros (participación, actividades constructivas, colaboración y motivación dentro del grupo), el tiempo de auto-estudio y los logros conseguidos (Kamp et al, 2012) .

\section{Desarrollo de la innovación}

Se analiza el desarrollo de la experiencia, mostrando en primer lugar las bases y objetivos del mismo, el plan de trabajo desarrollado y el diseño de actividades y programación empleado.

\subsection{Bases y objetivos}

El objetivo principal es favorecer el aprendizaje de las asignaturas de Construcción I y Economía del primer curso de Grado en Arquitectura Técnica mediante la transversalidad e interdisciplinariedad de las mismas, planteando los siguientes objetivos específicos:

- Seleccionar de los conocimientos, habilidades y actitudes adquiridos en la asignatura de Construcción I con el fin de aplicarlos directamente en las prácticas de Economía.

- Diseñar actividades conjuntas a realizar por los alumnos de ambas asignaturas que supongan el empleo y aprendizaje de los contenidos anteriormente descritos.

- Diseñar una programación adecuada y mecanismos de coordinación entre las asignaturas para que los conocimientos, habilidades y actitudes 
experimentados en Construcción I, sean inmediatamente aplicados en las prácticas de Economía, mediante las actividades integradoras anteriormente citadas.

- Generar de un valor añadido y diferenciador a nuestros alumnos aplicando el desarrollo tanto de las competencias específicas como de las denominadas dimensiones competenciales UPV.

Conseguidos los objetivos, se estima que el alumno mejorará el aprendizaje de la asignatura de Construcción I, estando más motivado y comprobando su aplicación práctica directa a través de las actividades propuestas en este proyecto. Del mismo modo, el aprendizaje en las prácticas de la asignatura de Economía les resultará más efectivo porque el alumno utilizará para su realización, las actividades integradoras propuestas en las asignaturas y los conocimientos adquiridos en Construcción I.

\subsection{Plan de trabajo}

Las asignaturas de Economía y Construcción I pertenecen a diferentes bloques de materias según el libro Blanco de la UPV del título del grado de ingeniería de edificación. No obstante, ambas asignaturas son de carácter de formación básica en la titulación que se imparte en el primer cuatrimestre del primer curso, lo que permite la transversalidad entre ambas. Con el fin de llevar a cabo el proyecto de innovación y mejora educativa se llevó a cabo el plan de trabajo donde se indicaron las siguientes tareas a desarrollar:

- Selección de los contenidos que se utilizarán en las actividades integradoras.

- Diseño de las actividades conjuntas.

- Planificación de las actividades.

- Evaluación de las actividades de Construcción I y Economía.

- Análisis y relación de los resultados de evaluación.

Estas tareas disponían de un responsable para su ejecución y reuniones semanales para el seguimiento del proyecto. Para la selección de los contenidos, cabe destacar que la asignatura de Economía consta de 7,5 European Credit Transfer Sistem (ECTS) y está dividida en dos grandes bloques según la guía docente (UPV, ETSIE 2015): Economía General y Gestión Empresarial en la edificación. Con el primer bloque el alumno conocerá el marco en el que se mueve la economía actual, a nivel básico, desde una óptica tanto microeconómica como macroeconómica. Con el bloque de Gestión Empresarial en la edificación, el alumno identificará las formas jurídicas de empresa, los requisitos legales para su constitución y los modelos de organización interna, estudiando los distintos departamentos que la pueden integrar. Además interpretará el patrimonio y los resultados de la empresa y será capaz de identificar distintas fuentes de financiación. Atendiendo a esto, el alumno debe realizar mediante unas prácticas de un Plan de creación de empresa donde se refunden ambos bloques de la asignatura. Estas prácticas constan de un total de 13 sesiones de 90 minutos.

Por otro lado, la asignatura de Construcción I consta de 9 ECTS donde el alumno debe conocer los elementos constructivos, su función, sus partes y forma de trabajo, tipologías de elementos constructivos y su técnica del proceso constructivo, conceptos de sostenibilidad y medio ambiente y el vocabulario técnico necesario para el mismo según marca la guía

(cc)) EY-NC-ND 2016, Universitat Politècnica de València 
docente (UPV, ETSIE 2015). En concreto, la selección y estructuración de las Unidades Didácticas es la siguiente:

Tabla 1: Unidades didácticas y temario de Construcción I

\begin{tabular}{|l|l|l|}
\hline & Unidades didácticas & Temas \\
\hline 1 & Introducción & Introducción al proceso constructivo \\
\hline 2 & El terreno y su acondicionamiento & $\begin{array}{l}\text { El terreno / Reconocimiento del terreno. Estudio Geotécnico / } \\
\text { Movimiento de tierras. Contención del terreno / Replanteos }\end{array}$ \\
\hline 3 & Elementos constructivos & $\begin{array}{l}\text { La cimentación / El muro / El arco / La bóveda / Estructura / } \\
\text { Forjados / Escalera / Cubierta }\end{array}$ \\
\hline 4 & Obras de fábrica & $\begin{array}{l}\text { Obras de fábrica. Generalidades / Obras de fábrica de piedra. } \\
\text { Mampostería y sillería / Fábricas de ladrillo / Fábricas de bloques }\end{array}$ \\
\hline 6 & Estructuras de madera & $\begin{array}{l}\text { La madera. Su utilización en la construcción de edificios. } \\
\text { Estructuras de madera }\end{array}$ \\
\hline
\end{tabular}

Tras mantener diferentes reflexiones por los profesores de ambas asignaturas se diseñó la actividad de realizar en las prácticas de la asignatura de Economía "El Plan de creación de empresa de una empresa de sector de la construcción" de forma que el alumno aplicara los conocimientos adquiridos en la asignatura Construcción I, es decir, el alumno puede generar cualquier empresa del sector de la construcción que recoja los conocimientos impartidos en Construcción I, como por ejemplo, una empresa de estructuras o estructuritas, de movimientos de tierras, de albañilería donde se desarrollan fábricas de ladrillo, cubiertas, escaleras, de derribos (demoliciones), de gestión de residuos y su tratamiento con el medio ambiente, etc. Así pues, con el fin de que el alumno sea capaz de demostrar la adquisición de los conocimientos y habilidades integradas de estas dos asignaturas fundamentales se llevó a cabo el PBL de forma interdisciplinar teniendo en cuenta los siguientes aspectos:

- Este proyecto se desarrollará únicamente en un grupo de los cinco existentes, de los alumnos matriculados en Economía y Construcción I.

- Este proyecto se realizará por equipos, con un alumno responsable que orga nice, gestione y planifique el tiempo del que se dispone para alcanzar sus objetivos comunes e individuales.

- El proyecto se presentará en un documento escrito y se expondrá de forma oral en el aula con el apoyo de una presentación del mismo.

Una vez diseñadas las actividades, se estableció de forma temporal la planificación de las mismas. Para ello, los profesores comunes a ese grupo debían tener una comunicación semanal que garantice el seguimiento del proyecto. Dado que los profesores comunes coincidían el mismo día de la semana de forma contigua en la impartición de clases del grupo seleccionado, se conjugaban 10 minutos de clase entre ambos profesores y los

(cc) BY-NC-ND 2016, Universitat Politècnica de València

Congreso In-Red (2016) 
alumnos seleccionados del proyecto. Por otro lado, en la asignatura de Economía el profesor supervisa la sesión para garantizar la estrategia, autonomía y flexibilidad del aprendizaje de forma que se les fomente de forma permanente $\mathrm{y}$, en la asignatura de Construcción I, se fomenta el uso de tutorías para la resolución de dudas después de cada sesión. El proyecto se entregará acompañado de un documento escrito que deberán comunicar oralmente en el aula. La evaluación por parte del profesorado se realizará en tres etapas:

Se valorará el análisis técnico-constructivo de los productos y servicios de la empresa constructora en la asignatura de Construcción I.

Se valorará la viabilidad técnica y económica del proyecto de la empresa constructora llevado a cabo en el proyecto, en la asignatura de Economía. Ambas valoraciones pasarán a una tercera etapa de evaluación donde se valorará la coherencia del producto o servicio de la empresa constructora con el análisis de viabilidad del proyecto de forma conjunta en las exposiciones de los proyectos por parte de ambas asignaturas.

\subsection{Diseño de actividades y programación}

En el presente curso 2015/2016 "El Plan de creación de empresa" de la asignatura de Economía se imparte durante todo el cuatrimestre estructurado en un total de 6 sesiones presenciales de 90 minutos de duración que corresponden al $20 \%$ de la nota de la asignatura de Economía. Dadas las condiciones desarrolladas en el apartado anterior, el alumno elaborará en equipo un Proyecto empresarial .La programación de las distintas sesiones se realizó según muestra la tabla 2.

Tabla 2: Planificación de sesiones de las prácticas "Plan de creación de Empresa"

\begin{tabular}{|c|c|c|c|}
\hline Sesión & Objetivos & $\begin{array}{ll}\text { Actividades } & \text { de } \\
\text { Construcción } & \end{array}$ & Actividades de Economía \\
\hline 1 & $\begin{array}{l}\text { Los estudiantes deben ser capaces } \\
\text { de seleccionar y argumentar una } \\
\text { Idea de Negocio del sector de la } \\
\text { Construcción }\end{array}$ & $\begin{array}{l}\text { Lluvia de ideas de } \\
\text { empresas de construcción } \\
\text { relacionadas con el } \\
\text { temario impartido. } \\
\text { Definición y argumento } \\
\text { de las posibles ideas. }\end{array}$ & $\begin{array}{l}\text { Clase teórico-práctica de los objetivos, } \\
\text { utilidades y criterios del Plan de Empresa. La } \\
\text { idea de Negocio. Explicación de la posibilidad } \\
\text { de acogerse al aprendizaje transversal con el } \\
\text { Profesor de Materiales de Construcción. }\end{array}$ \\
\hline 2 & $\begin{array}{l}\text { Los estudiantes deben elaborar el } \\
\text { Plan de Gestión de su plan de } \\
\text { empresa. }\end{array}$ & $\begin{array}{l}\text { Identificación de los } \\
\text { trabajos necesarios para } \\
\text { llevar cabo la empresa } \\
\text { seleccionada y personal } \\
\text { necesario. }\end{array}$ & $\begin{array}{l}\text { Clase teórico-práctica del Plan de Gestión de la } \\
\text { empresa. Esta recoge la forma jurídica óptima } \\
\text { para la empresa, análisis DAFO, Plan } \\
\text { Organizativo, de Gestión y Recursos humanos. }\end{array}$ \\
\hline 3 & $\begin{array}{l}\text { Los estudiantes deben analizar el } \\
\text { mercado al que corresponde el } \\
\text { producto o servicio ofertado por su }\end{array}$ & 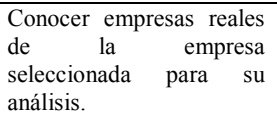 & $\begin{array}{l}\text { Clase teórico-práctica de Análisis del Mercado } \\
\text { que comprende el mercado, producto o servicio, } \\
\text { competencia, clientela y proveedores. }\end{array}$ \\
\hline 4 & $\begin{array}{l}\text { Los estudiantes deben ser capaces } \\
\text { de elaborar el Plan de Marketing. }\end{array}$ & $\begin{array}{l}\text { Análisis de Marketing de } \\
\text { empresas similares a la } \\
\text { seleccionado. }\end{array}$ & $\begin{array}{l}\text { Clase teórico-práctica del Plan de Marketing } \\
\text { centrado en la aplicación de Marketing Mix a } \\
\text { tan solo uno de los productos ofertados dirigido } \\
\text { a un segmento objetivo. }\end{array}$ \\
\hline 5 & $\begin{array}{l}\text { Los estudiantes deben ser capaces } \\
\text { de analizar los objetivos de ventas y } \\
\text { crédito necesario para que la } \\
\text { empresa sea } \\
\text { económicamente. viable }\end{array}$ & $\begin{array}{l}\text { Clase teórico-práctica del } \\
\text { Plan de Económico- } \\
\text { Financiero de la empresa. } \\
\text { Objetivos de Ventas, } \\
\text { Pagos y Préstamo. }\end{array}$ & $\begin{array}{l}\text { Conocer cómo se establecen los objetivos de } \\
\text { ventas en la empresa seleccionada (unidades, } \\
\text { toneladas, } \mathrm{m}^{2}, \mathrm{~m}^{3} \ldots \text { ) }\end{array}$ \\
\hline
\end{tabular}

(cc)) EY-NC-ND 2016, Universitat Politècnica de València 
\begin{tabular}{|l|ll|l|}
\hline 6 & Los estudiantes ser capaces de & Exposición oral.
\end{tabular}

transmitir su plan de empresa y captar la atención.

La evaluación por parte de la asignatura de Construcción I corresponde a un 15\% de la nota del plan de empresa realizado.

\section{Resultados}

El grupo de prácticas seleccionado para llevar a cabo la innovación docente mediante la metodología ABP estaba conformado por un total de 20 alumnos de 72 alumnos en total matriculados en ambas asignaturas. Así mismo, como la realización era en grupo de entre 3 y 4 componentes se formaron 5 grupos de 4 componentes que llevaron a cabo la transversalidad entre las prácticas de Economía y Construcción I.

En concreto, se han realizado los Planes de empresa del sector de la construcción que se recogen a continuación en la Fig. 1:

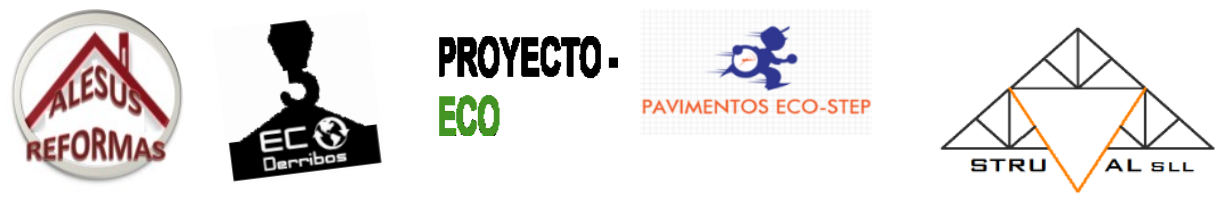

Fig. 1 Planes de empresa del sector de la construcción

Como se puede identificar en los logotipos, la empresa Alesus Reformas trata los conceptos de la asignatura Construcción I de obras de fábrica, EC Derribos recoge principalmente el tema de demoliciones y su gestión medioambiental, Proyecto ECO es una empresa destinada a la rehabilitación de inmuebles para la mejora energética de edificios con lo que recoge conceptos de la asignatura tales como obras de fábrica, procesos de edificación y gestión medioambiental, Pavimentos ECO-STEP trabaja sobre la disposición de pavimentos que recogen energía tratando temas medioambientales. Por último, Struval es una empresa que diseña y ejecuta estructuras tanto espaciales como de hormigón armado trabajando con los temas de replanteo, cimentaciones y estructuras. De este modo, se constata que la innovación pedagógica en estas dos asignaturas muestra una interdisciplinariedad entre ambas en función de las empresas elegidas por los alumnos. Además, las notas resultantes del grupo (A) que realizó la innovación han sido más positivas que en los restantes grupos que no realizaron la misma. Como puede verse en el Tabla 3, la nota media de este grupo supera en más de 6 décimas del promedio en las prácticas de economía de todos los grupos.

Tabla 3: Nota media por grupo de la realización del "Plan de Empresa"

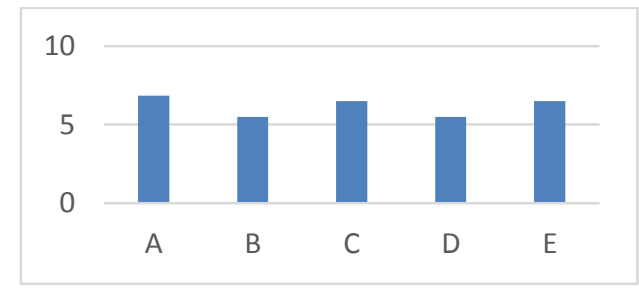

(cc) EY-NC-ND 2016, Universitat Politècnica de València

Congreso In-Red (2016) 
En definitiva, los resultados no solo muestran la metodología implantada ha facilitado la interdisciplinariedad de los conocimientos de ambas materias, sino que ha permitido integración de los diferentes conocimientos adquiridos en las dos asignaturas de forma sobresaliente.

\section{Conclusiones}

El desarrollo del PIME (A02/15) ha generado un gran reto tanto a los alumnos como a los profesores de las asignaturas de Construcción I y Economía. De hecho, este proyecto ha necesitado de una gran vinculación y coordinación entre los docentes para llegar al éxito del mismo, suponiendo un mayor esfuerzo que el esperado. Según los resultados obtenidos corroboran que existe una mayor autoeficacia gracias a la metodología empleada y una mejor familiaridad ante el conocimiento del problema gracias a la interdisciplinariedad existente en este proyecto. En conclusión, gracias a esta iniciativa se ha generado un valor añadido y diferenciador a nuestros alumnos que les permite relacionar conocimientos, habilidades y actitudes de distintas disciplinas. Es importante destacar que existen diferentes limitaciones debido al poco tiempo que se posee para desarrollar profundamente cada uno de los aspectos ante la diversidad de los planes de empresa empleados. Es por esto, para futuras líneas de trabajo, sería conveniente que un mismo grupo desarrollara una misma idea de negocio con el fin de realizar una puesta en común de dudas y problemas.

Por último, nos gustaría destacar que se ha observado un grado de motivación superior en los alumnos que han realizado la innovación mostrando su interés en el aula y en la exposición final de su trabajo.

\section{Referencias}

DUNLAP, Joanna C. (2005) "Problem-based learning and self-efficacy: How a capstone course prepares students for a profession". Educational Technology Research and Development. Springer, Vol. 53. Num. 1, p.65-83.

HMELO-SILVER, Cindy (2004) "Problem-Based Learning: What and how do Students Learn?" Educational Psychology Review. Springer Journals, Vol. 16, Num. 3, p.235.

KAMP,Rachelle J. A. ,DOLMANS,Diana H. J. M. ,BERKEL,Henk J. M. van ,SCHMIDT,

Henk G. (2012) “The relationship between students' small group activities, time spent on self-study, and achievement" Higher Education, Volume 64, Issue 3, pp 385-397.

SOCKALINGAM, Nachamma, ROTGANS, Jerome I., SCHMIDT, Henk G. (2011) "The relationships between problem characteristics, achievement-related behaviors, and academic achievement in problem-based learning" Advances in Health Sciences Education, Vol. 16. Num. 4, p.481-490.

SOCKALINGAM, Nachamma, SCHMIDT, Henk G. (2013) "Does the extent of problem familiarity influence students' learning in problem-based learning?" Instructional Science, Volume 41, Issue 5, pp 921-932.

UPV, EICE (2015): Equipos de innovación y calidad educativa (EICE).

$<$ http://www.upv.es/contenidos/EQIN/indexc.html> [Consulta: 8 de enero de 2016].

UPV, ETSIE (2015): Equipos de innovación y calidad educativa (EICE).

$<$ http://www.upv.es/titulaciones/GIE/menu_920670c.html > [Consulta: 10 de enero de 2016].

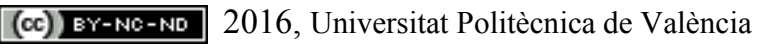

Congreso IN-RED (2016) 